\title{
Arritmia Ventricular Potencialmente Fatal Induzida por Taquicardia Atrial em uma Criança com Mutação de SCN5A
}

\author{
Life-Threatening Ventricular Arrhythmia Induced by Atrial Tachycardia in a Child with an SCN5A Mutation
}

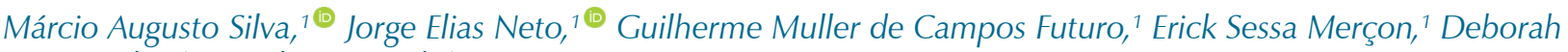
Vasconcelos, ${ }^{1}$ Ricardo Kuniyoshi ${ }^{1}$

Vitoria Apart Hospital - Cardiologia, ${ }^{1}$ Serra, ES - Brasil

\section{Introdução}

Mutações no gene SCN5A, que codifica o canal $\mathrm{Na}+$ cardíaco, podem causar arritmias potencialmente fatais. Identificou-se que essas mutações são causadoras de doenças elétricas primárias hereditárias, incluindo a síndrome de Brugada (SB), síndrome do QT longo, e outros distúrbios da condução cardíaca. ${ }^{1,2}$ A SB, a doença mais relatada desse grupo de transtornos, geralmente é descrita em populações adultas e está relacionada a aproximadamente $20 \%$ de todas as mortes súbitas (MS) em pacientes com corações aparentemente normais. ${ }^{3-5}$ Portanto, alguns relatos mostraram eventos arrítmicos (EA) significativos causados por essa mutação na infância. ${ }^{6}$

Arritmias atriais, bem como a síndrome do nó sinusal (SNS), podem estar relacionadas a anormalidades do canal $\mathrm{Na}+$. Na SB, arritmias atriais são diagnosticadas em até 38\% dos pacientes, e estão relacionadas a prognósticos piores. ${ }^{7}$

\section{Relato de Caso}

Um menino de 2 anos de idade, sem anomalias cardíacas detectadas em exames de ecocardiograma transtorácico e imagens de ressonância magnética (RM), deu entrada duas vezes no hospital com padrão típico de flutter atrial (FLA), que foi revertido por cardioversão elétrica. Após o último episódio, ele recebeu alta com prescrição de $3 \mathrm{mg} / \mathrm{kg}$ de amiodarona por dia. O ECG de 12 derivações revelou intervalos QT (390 $410 \mathrm{~ms}$ ) e QTc (413 - $440 \mathrm{~ms}$ ) normais, intervalo PR (200 ms) prolongado, e ondas $\mathrm{T}$ negativas nas derivações precordiais direitas (Figura 1).

Um Holter de 24 horas, obtido três meses mais tarde, depois da suspensão da amiodarona, registrou um episódio sincopal - respiração agônica, cianose, movimentos convulsivos - durante o sono no colo de sua mãe. A faixa de ECG apresentou uma ampla variação de ciclo RR, com

\section{Palavras-chave}

Arritmias Cardíacas; Síndrome de Brugada; Criança.

Correspondência: Márcio Augusto Silva •

Vitoria Apart Hospital - Cardiologia - Rodovia BR 101 Norte Km 2. CEP

29161-001, Boa Vista, Serra, ES - Brasil

E-mail: masilva.cardio@gmail.com

Artigo recebido em 21/05/2020, revisado em 08/09/2020, aceito em $04 / 11 / 2020$

DOI: https://doi.org/10.36660/abc.20200509 episódios intermitentes de taquicardia atrial (TA), que se tornou sustentada de condução atrioventricular (AV) de 1:1, com alargamento progressivo do complexo QRS, seguido de taquicardia ventricular (TV) polimórfica, fibrilação ventricular (FV) e 30 segundos de assistolia; o ritmo sinusal foi restaurado espontaneamente (Figura 2).

Houve uma nova ocorrência de taquicardia com QRS alargado na unidade de terapia intensiva. A infusão de adenosina $1 \mathrm{mg}$ nesse momento revelou um novo FLA antes de uma nova cardioversão elétrica.

Estudo eletrofisiológico (EEF) foi realizado sob sedação intravenosa profunda (quetamina 0,2 mg/kg e infusão contínua de propofol) com dois cateteres multipolares 5F. Detectouse um aumento do intervalo HV (63 ms), e FLA típico, dependente do istmo cavo tricuspídeo (ICT) foi induzido por estimulação atrial programada. Foi realizada ablação linear por cateter de radiofrequência (RF) com bloqueio bidirecional da condução pelo ICT. A estimulação programada (2 ciclos e 2 extra-estímulos) não induziu arritmias ventriculares.

Testes genéticos identificaram uma mutação patogênica heterozigótica do gene SCN5A - variante c.362G>A p. (Arg121Gln) no éxon 3 do gene SCN5A (NM_198056), compatível com a SB.

O paciente é filho único, sem histórico familiar de arritmias, síncope ou MS. A mãe, de 30 anos de idade, apresentou ECG normal e painel genético negativo. O pai, de 34 anos e assintomático, apresentou um bloqueio AV de primeiro grau (PR = 220 ms) e padrão típico de SB Tipo I no ECG (Figura 1), intervalo HV anormal (73 ms), ausência de arritmias ventriculares induzíveis e mesma mutação do gene SCN5A.

Depois de 18 meses de acompanhamento sem sintomas ou EA, apresentou um novo episódio sincopal, desencadeado por febre. Não foram observadas alterações eletrocardiográficas, tanto no ECG de repouso como no Holter de 24 horas. Nesse momento, foi implantado um cardioversor desfibrilador implantável (CDI) de câmara única por via transvenosa. No acompanhamento de 6 meses, não foram observadas complicações relacionadas ao dispositivo, EA ou terapias do CDI.

\section{Discussão}

O presente estudo relatou o caso de um menino de 2 anos com um coração aparentemente normal com EA potencialmente fatal desencadeada por TA sustentada. Esse evento incomum nos levou a suspeitar de uma possível canalopatia. Foi detectada mutação no SCN5A na criança e em seu pai, que apresentou um padrão de SB em ECG Tipo I. 

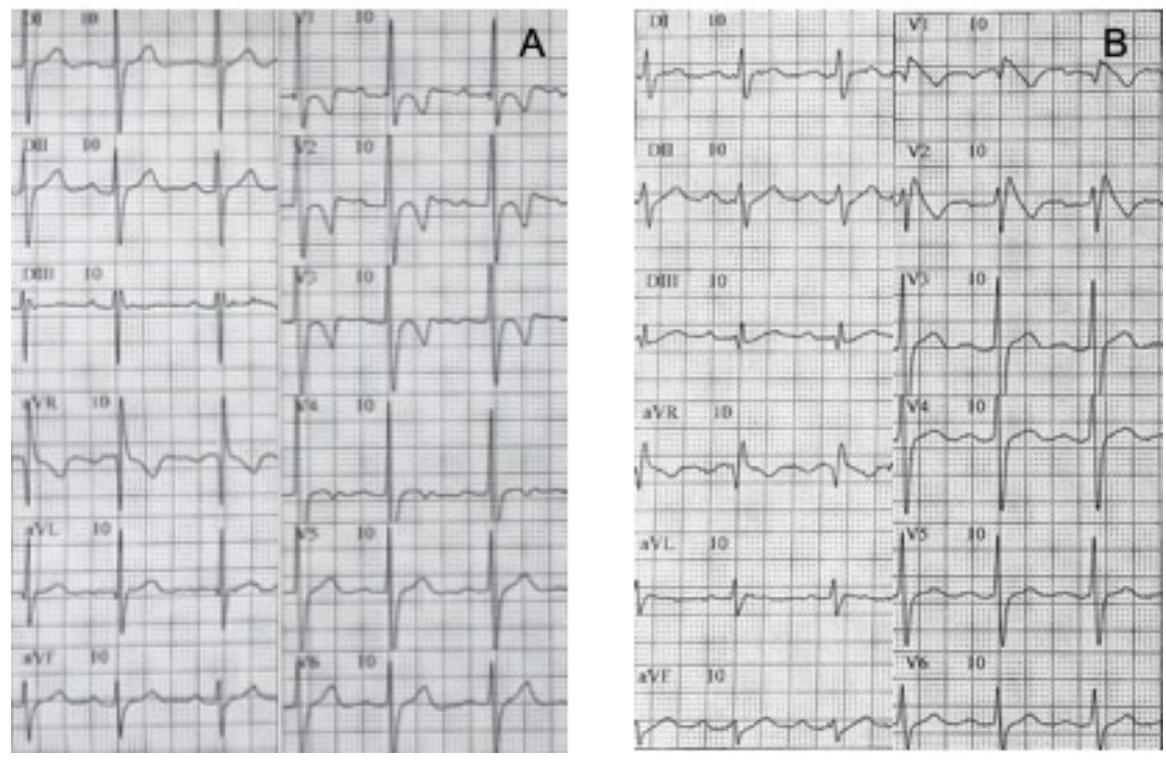

Figura 1 - ECG de 12 derivações espontâneas (A) - Criança; (B) - Pai.


G
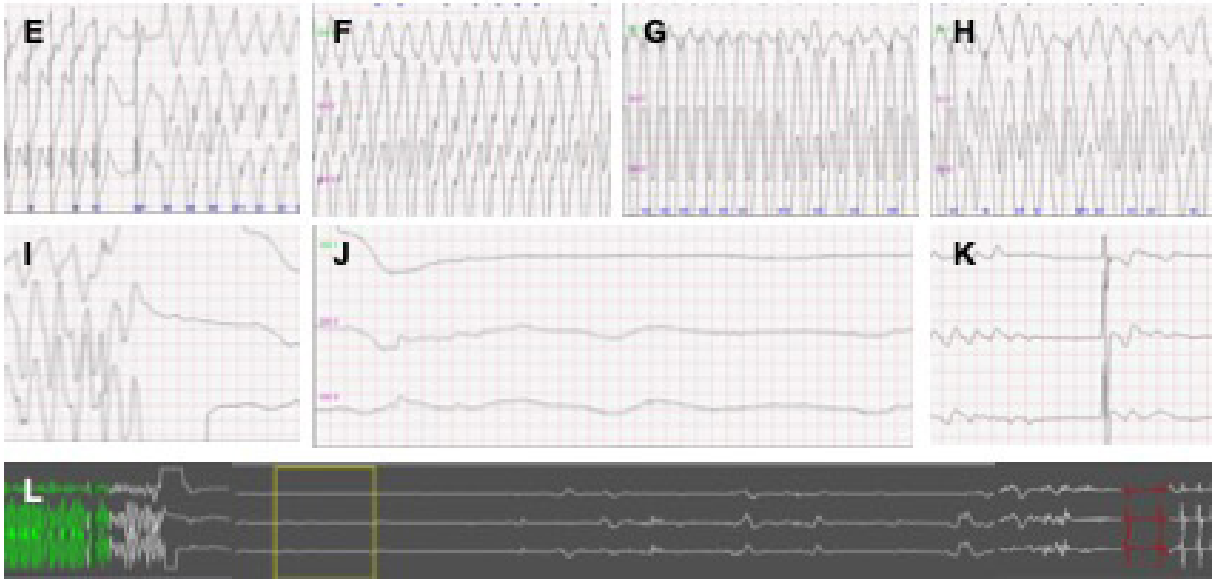

Figura 2 - Evento sincopal registrado no Holter de 24 horas. A, B - TA/AFL de condução AV variável, com QRS estreito; C, D - A condução AV passou a 1:1 com alargamento progressivo de QRS; E, F, G, H - TF e TV polimórfica ampla sustentada; I, J, K - a FV é seguida de 30 segundos de assistolia, com recuperação espontânea do ritmo sinusal; $L$ - faixa longa de assistolia total. 
Desde a descrição inicial da doença em 1992, que incluiu três crianças, ${ }^{3}$ os dados sobre a população pediátrica com SB publicados são muito limitados. O padrão de ECG típico da SB (tipo I - elevação côncava do ST nas derivações precordiais direitas) e as manifestações clínicas geralmente não são observadas em crianças mais novas. A idade do aparecimento dos sintomas e EA varia entre 40 e 50 anos, e eles são raros em crianças e idosos. ${ }^{5} \mathrm{Na}$ SABRUS (Survey on Arrhythmic Events in Brugada Syndrome - Pesquisa sobre Eventos Arrítmicos na Síndrome de Brugada), que incluiu 678 pacientes com SB, a grande maioria (94,2\%) dos pacientes tinha entre 16 e 70 anos no momento do primeiro EA, enquanto pacientes pediátricos (<16 anos) e idosos ( $>70$ anos) representavam $4,3 \%$ e $1,5 \%$, respectivamente. ${ }^{8}$ A síncope geralmente é a primeira manifestação clínica em 14 a 21\%, e a MS acontece em 5 a $7 \%$ dos pacientes pediátricos com SB, mas a maioria é assintomática. ${ }^{9,10} \mathrm{~A}$ predominância significativa de pacientes do sexo masculino existente entre adultos não é observada em crianças na pré-puberdade, possivelmente devido a influências hormonais, especialmente os níveis de testosterona. ${ }^{5,11}$

Em nosso paciente, o EA ocorreu durante o sono, como é frequentemente descrito em pacientes com SB, o que sugere uma associação com bradicardia e possivelmente modulação vagal. No presente caso, o evento arrítmico sincopal registrado no Holter de 24 horas - TV/FV - foi desencadeado por uma TA com condução AV rápida durante o sono, sugerindo alguma influência vagal. Sabe-se também que a febre comumente desencadeia arritmias (e pode revelar o padrão típico de ECG), ${ }^{6,12}$ e ela foi observada no segundo evento sincopal do paciente deste estudo. Há alguns relatos na literatura de arritmias potencialmente fatais e MS em pacientes muito jovens com SB, mas nenhum claramente documentou uma participação direta de uma TA na indução de TV/FV.

A estratificação de risco em pacientes jovens ainda continua a ser um desafio. O padrão de ECG tipo I, síncope, MS, disfunção do nó sinusal, arritmias atriais, anormalidades na condução, e arritmias ventriculares induzidas em EEF foram descritos como preditores de eventos potencialmente fatais., ${ }^{9,10}$

Nenhum estudo amplo comprovou que a presença de uma mutação do SCN5A é um marcador de risco. Entretanto, mutações complexas do SCN5A parecem levar fenótipos mais graves. ${ }^{13}$

Embora essa criança, desde o evento inicial, tenha atendido aos critérios para CDI, os riscos potenciais de um CDI em crianças muito jovens (terapias inapropriadas e complicações relacionadas aos eletrodos) foram levados em consideração na decisão de adiar o implante. Além disso, a possibilidade da ablação da arritmia desencadeadora de FV (FLA) nos levou a acreditar em uma chance menor de recorrência precoce.

\section{Conclusão}

O caso apresentado demonstra uma apresentação grave de arritmia numa criança, que foi diagnosticada com uma mutação no gene SCN5A. O circuito desencadeador de TV/ FV - flutter atrial - foi eliminado por ablação, resultando em alívio dos sintomas por um longo período, mas o implante CDI foi realizado devido à recorrência da síncope. Isso reforça o quão complexa pode ser a apresentação e a evolução de algumas canalopatias na população pediátrica.

\section{Contribuição dos autores}

Concepção e desenho da pesquisa e Análise e interpretação dos dados: Silva MA; Obtenção de dados: Silva MA, Elias Neto J, Futuro GMC, Merçon ES, Vasconcelos D, Kuniyoshi R; Redação do manuscrito: Silva MA, Elias Neto J; Revisão crítica do manuscrito quanto ao conteúdo intelectual importante: Silva MA, Elias Neto J, Futuro GMC, Merçon ES.

\section{Potencial conflito de interesse}

Não há conflito com o presente artigo

\section{Fontes de financiamento}

O presente estudo não teve fontes de financiamento externas.

\section{Vinculação acadêmica}

Não há vinculação deste estudo a programas de pósgraduação.

\section{Aprovação ética e consentimento informado}

Este artigo não contém estudos com humanos ou animais realizados por nenhum dos autores.

\section{Referências}

1. Zaklyazminskaya E, Dzemeshkevich S. The Role of Mutations in the SCN5A Gene in Cardiomyopathies. Biochim Biophys Acta. 2016;1863(7 Pt B):1799-805. doi: 10.1016/j.bbamcr.2016.02.014.

2. Remme CA. Cardiac Sodium Channelopathy Associated with SCN5A Mutations: Electrophysiological, Molecular and Genetic Aspects. J Physiol. 2013;591(17):4099-116. doi: 10.1113/jphysiol.2013.256461.

3. Brugada P, Brugada J. Right Bundle Branch Block, Persistent ST Segment Elevation and Sudden Cardiac Death: A Distinct Clinical and Electrocardiographic Syndrome. A Multicenter Report. J Am Coll Cardiol. 1992;20(6):1391-6. doi: 10.1016/0735-1097(92)90253-j.

4. Brugada J, Campuzano O, Arbelo E, Sarquella-Brugada G, Brugada R. Present Status of Brugada Syndrome: JACC State-of-the-Art Review. J Am Coll Cardiol. 2018;72(9):1046-1059. doi: 10.1016/j. jacc.2018.06.037.

5. Brugada P. Brugada syndrome: More than 20 Years of Scientific Excitement. J Cardiol. 2016;67(3):215-20. doi: 10.1016/j.jjcc.2015.08.009.

6. Chockalingam P, Rammeloo LA, Postema PG, Hruda J, Clur SA, Blom NA, et al. Fever-induced Life-threatening Arrhythmias in Children Harboring an SCN5A Mutation. Pediatrics. 2011;127(1):e239-44. doi: 10.1542/ peds.2010-1688. 
7. Francis J, Antzelevitch C. Atrial Fibrillation and Brugada Syndrome.J Am Coll Cardiol. 2008;51(12):1149-53. doi: 10.1016/j.jacc.2007.10.062.

8. Milman A, Andorin A, Gourraud JB, Sacher F, Mabo P, Kim SH, et al. Age of First Arrhythmic Event in Brugada Syndrome: Data From the SABRUS (Survey on Arrhythmic Events in Brugada Syndrome) in 678 Patients. Circ Arrhythm Electrophysiol. 2017;10(12):e005222. doi: 10.1161/CIRCEP.117.005222.

9. Corcia MCG, Sieira J, Pappaert G, Asmundis C, Chierchia GB, Sarkozy A, et al. A Clinical Score Model to Predict Lethal Events in Young Patients ( $\leq 19$ Years) With the Brugada Syndrome. Am J Cardiol. 2017;120(5):797-802. doi: 10.1016/j.amjcard.2017.05.056.

10. Andorin A, Behr ER, Denjoy I,Crotti L, Dagradi F, Jesel L, etal. Impact of Clinical and Genetic Findings on the Management of Young Patients with Brugada Syndrome. Heart Rhythm. 2016;13(6):1274-82. doi: 10.1016/.jhrthm.2016.02.013.
11. Mariani S, Musumeci B, Basciani S, Fiore D, Francia P, Persichetti A, et al. Lack of Influence of the Androgen Receptor Gene CAG-Repeat Polymorphism on Clinical and Electrocardiographic Manifestations of the Brugada Syndrome in Man. Clin Med Insights Cardiol. 2012;6:145-52. doi: 10.4137/CMC.S10553.

12. Dumaine R, Towbin JA, Brugada P, Vatta M, Nesterenko DV, Nesterenko $\mathrm{VV}$, et al. Ionic Mechanisms Responsible for the Electrocardiographic Phenotype of the Brugada Syndrome are Temperature Dependent. Circ Res. 1999;85(9):803-9. doi: 10.1161/01.res.85.9.803.

13. Sacilotto L, Epifanio HB, Darrieux FC, Wulkan F, Oliveira TG, Hachul DT, et al. Compound Heterozygous SCN5A Mutations in a Toddler Are they Associated with a More Severe Phenotype? Arq Bras Cardiol. 2017;108(1):70-73. doi: 10.5935/abc.20170006. 